\title{
Second-Generation Antipsychotic Discontinuation in First Episode Psychosis: An Updated Review
}

\author{
Brian J. Miller, Chelsea Bodenheimer, Krystle Crittenden \\ Department of Psychiatry and Health Behavior, Georgia Health Sciences University, Augusta, GA, USA
}

\begin{abstract}
"All-causes discontinuation" refers to discontinuation of treatment for any reason, and medication adherence is an important component of this measure. Similar to our previous results, we found that almost $30 \%$ of patients with first-episode psychosis (FEP) discontinue medication in the first 9 months of treatment, a finding that has important implications for long-term outcomes. Many newer second-generation antipsychotics have not been studied in FEP. The self-reported Drug Attitude Inventory may help identify patients at heightened risk for medication discontinuation. In addition to vigilant monitoring for and adequate treatment of psychopathology and medication side effects, Relapse Prevention Therapy and the use of long-acting injectable agents may be effective interventions decrease discontinuation rates in FEP. There is currently no consensus on how long a patient should remain on an antipsychotic medication following remission of FEP. Studies are needed to identify predictors of which patients in remission from FEP are less likely to relapse when medication is discontinued. Taken together, our findings presented here underscore the importance of addressing medication discontinuation both as a means of preventing long-term morbidity and enhancing remission and functional recovery in FEP.
\end{abstract}

KEY WORDS: Schizophrenia; Antipsychotic agents; Patient nonadherence; Epidemiology.

\section{INTRODUCTION}

In recent years, there has been a tremendous growth in research on first-episode psychosis (FEP). The initial treatment of FEP represents a critical juncture that has the potential to shape patient and family attitudes about subsequent treatment, as well as the longitudinal course and outcome of illness. The term all-causes discontinuation is defined as discontinuation of treatment for any reason, including lack of efficacy, intolerable side effects or other adverse effects, and patient and/or clinician decision to stop medication altogether or switch to a different medication. Thus, medication adherence is an important component of this measure.

Three landmark trials published in the past five years have brought the issue of all-causes medication discontinuation in patients with FEP to the forefront of clinical attention. In a two-year study of 263 patients with

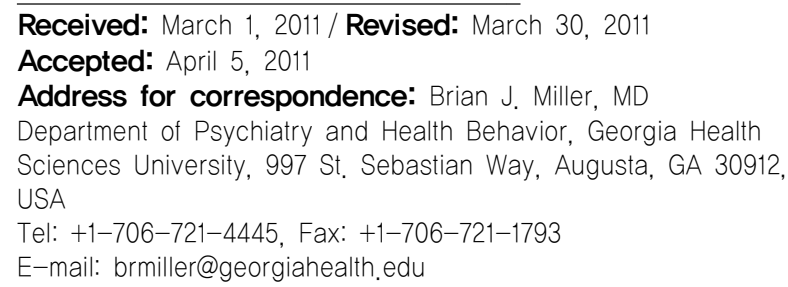

FEP, Green et al. ${ }^{1)}$ reported a $76.6 \%$ all-causes discontinuation rate in patients randomized to olanzapine, and $30 \%$ of discontinuations were due to "patient decision". Secondly, in the Comparison of Atypicals in First Episode (CAFE) study of 400 patients with FEP, McEvoy et al. $^{2)}$ reported a $70 \%$ all-causes discontinuation rate, and over half of the discontinuations occurred against the recommendations of the treating clinicians. Third, Kahn et $a l^{3)}$ reported a $42 \%$ all-causes discontinuation rate among the 498 patients in the European First Episode Schizophrenia Trial (EUFEST). These three studies suggest that medication discontinuation is a major issue in FEP from the onset of treatment, with a prevalence comparable to that observed in the Clinical Antipsychotic Trials of Intervention Effectiveness (CATIE) study, ${ }^{4}$ the single largest treatment study of patients with chronic schizophrenia. We previously found a $40.8 \%$ all-causes discontinuation rate in a review of 28 studies of second-generation antipsychotics (SGAs) in FEP published between 1997 and 2008, ${ }^{5}$ which was consistent with the high prevalence of this phenomenon observed in these three large trials.

Since our original review, ${ }^{5)}$ the 2009 update of the Schizophrenia Patient Outcomes Research Team (PORT) 
psychopharmacological treatment recommendations and summary statements was published (Buchanan et al. ${ }^{6}$ ). Two of the sixteen PORT treatment recommendations were specific to FEP. Furthermore, in the past three years, there have been several new clinical trials of SGAs in FEP, including agents and formulations not covered in our previous review, as well as several newly approved SGAs for the treatment of schizophrenia. The purpose of this paper is to provide an update on all-causes discontinuation of SGAs in FEP. We will first review current treatment recommendations for FEP. Next, we will describe the prevalence of all-causes discontinuation in studies of SGAs in FEP published since our original review. We will then discuss reasons and risk factors contributing to medication discontinuation. Lastly, we will explore potential interventions to decrease discontinuation rates.

\section{PHARMACOLOGIC TREATMENT OF FEP}

In the 2006 update of the Texas Medication Algorithm Project (TMAP), ${ }^{7)}$ the majority opinion was that SGAs (versus first-generation antipsychotics [FGAs]) are preferred for the treatment of FEP (and for patients never before treated with an SGA), although a particular "lead-off" agent was not preferentially endorsed. There was also a general consensus recommendation from the TMAP that patients with FEP typically require lower antipsychotic doses-on average about half of the dose needed to treat chronic schizophrenia-although quetiapine was noted to be a possible exception. However, while patients with FEP are noted to have higher positive symptom response rates than patients with chronic schizophrenia, they may also be particularly sensitive to side effects, including metabolic changes, weight gain, and extrapyramidal symptoms.

Similar to the TMAP, the 2009 PORT recommended that patients with FEP should be treated with lower doses of SGAs than those use for chronic schizophrenia, owing to both increased treatment responsiveness and increased sensitivity to adverse side effects. ${ }^{6}$ Quetiapine was noted to be an exception, with a recommended titrated dose of $500-600 \mathrm{mg} /$ day, and no data were available to evaluate the efficacy of low-dose aripiprazole or ziprasidone in FEP. In contrast to the TMAP, the PORT recommended antipsychotic medications other than clozapine or olanzapine as first-line treatment for FEP with acute positive symptoms, citing a lack of clinically significant differences in efficacy between SGAs and FGAs. Clozapine was not recommended as first-line treatment of FEP based on a 52-week trial that found negligible differences in efficacy between clozapine and chlorpromazine, ${ }^{8)}$ coupled with its adverse side effect profile. In several "headto-head" trials of different SGAs, no clinically meaningful differences in efficacy have been observed. Thus, as treatment with olanzapine has consistently been associated with the greatest liability to weight gain and other components of the metabolic syndrome, this agent was not recommended as first-line treatment of FEP.

\section{PREVALENCE OF SGA DISCONTINUATION IN RECENT STUDIES OF FEP}

We report here the prevalence of all-causes discontinuation in studies of SGAs in FEP-defined as schizophrenia and other nonaffective psychoses-published since our original review. We have chosen to restrict our review to SGAs based on (1) treatment recommendations from the TMAP and PORT, as described above, (2) several newly-approved SGAs for the treatment of schizophrenia since our previous review, (3) a paucity of recent studies of FGAs in FEP, and (4) several studies which found greater long-term benefits for the use of SGAs (versus FGAs) in terms of higher remission rates ${ }^{1,9)}$ and a lower prevalence of medication discontinuation. ${ }^{1,3)}$

A systematic review of the literature was performed to identify studies published since 2008 that investigated the efficacy of SGAs in FEP and reported data on all-causes discontinuation. Potential studies were identified in PubMed using the following search strategy: "first-episode psychosis or first-episode schizophrenia" and "risperidone or risperdal", restricting to clinical trials in humans published in the last three years. This search strategy was repeated for amisulpiride, aripiprazole, asenapine, iloperidone, lurasidone, olanzapine, paliperidone, quetiapine, and ziprasidone. The reference lists of identified studies were also thoroughly reviewed. We excluded studies that were included in our previous review. A total of 9 studies, 5 that investigated single SGA agents, ${ }^{10-14)}$ and 4 that compared multiple SGAs ${ }^{15-18)}$ were identified. The general characteristics of these studies are outlined in Table 1.

The 9 included studies involved a total of 648 patients. Data were stratified based on individual SGAs; for comparative purposes, oral risperidone and long-acting injectable risperidone were considered separately. These trials included data from 1 study of aripiprazole, 1 study of olanzapine, 3 studies of quetiapine, 5 studies of oral risperidone, and 3 studies of risperidone long-acting injec- 
Table 1. Recent clinical studies of SGAs in FEP

\begin{tabular}{|c|c|c|c|c|c|c|c|c|}
\hline \multirow[t]{2}{*}{ SGA } & \multirow[t]{2}{*}{ Study } & \multirow[t]{2}{*}{ Type of trial } & \multirow{2}{*}{$\begin{array}{l}\text { Duration } \\
\text { (Week) }\end{array}$} & \multirow{2}{*}{$\begin{array}{c}\text { Subjects } \\
\mathrm{N}\end{array}$} & \multirow{2}{*}{$\begin{array}{c}\text { Mean } \\
\text { dose }\end{array}$} & \multicolumn{2}{|c|}{$\begin{array}{c}\text { All-causes } \\
\text { discontinuation }\end{array}$} & \multirow[t]{2}{*}{ Comparator } \\
\hline & & & & & & $\mathrm{N}$ & $\%$ & \\
\hline Aripiprazole & Takahashi et al. $^{14)}$ & Open-label & 12 & 45 & 17.8 & 5 & 11.1 & None \\
\hline Amisulpiride & Kahn et al. $^{3)}$ & Open-label & 52 & 104 & 450.8 & 32 & 30.8 & Multiple \\
\hline Asenapine & None & & & & & & & \\
\hline lloperidone & None & & & & & & & \\
\hline Lurasidone & None & & & & & & & \\
\hline Olanzapine & Cuesta et $a l^{15)}$ & RCT, Open-label & 26 & 44 & 10.8 & 9 & 20.5 & Risperidone \\
\hline \multirow{4}{*}{ Quetiapine } & $\begin{array}{l}\text { None } \\
\text { Andersen et } \text { al. }^{10)}\end{array}$ & Open-label & 26 & $\Delta 6$ & & & & \\
\hline & Berger et al. ${ }^{11)}$ & RCT, Double-blinded & $\begin{array}{l}20 \\
12\end{array}$ & $\begin{array}{r}46 \\
134\end{array}$ & $\begin{array}{l}519.6 \\
301.6\end{array}$ & $\begin{array}{l}22 \\
40\end{array}$ & $\begin{array}{l}47.8 \\
299\end{array}$ & $\begin{array}{l}\text { None } \\
\text { Seroquel (200 vs. } 400 \text { ma) }\end{array}$ \\
\hline & Gafoor et al. ${ }^{16)}$ & RCT, Open-label & 12 & 38 & 375.0 & 28 & $\begin{array}{l}29.4 \\
73.7\end{array}$ & $\begin{array}{l}\text { Risperidone } \\
\text { Rid } 400 \mathrm{mg})\end{array}$ \\
\hline & Mean/Total & & 17 & 218 & 398.7 & 90 & 50.5 & \\
\hline \multirow[t]{6}{*}{ Risperidone } & Cuesta et al. ${ }^{15)}$ & RCT, Open-label & 26 & 56 & 6.1 & 12 & 21.4 & Olanzapine \\
\hline & Gafoor et $a l^{16)}$ & RCT, Open-label & 12 & 34 & 2.7 & 19 & 55.9 & Olanzapine \\
\hline & Kim et $a l^{17)}$ & RCT, Open-label & 104 & 30 & 2.8 & 2 & 6.7 & Risperidone LAl \\
\hline & Möller et al. ${ }^{13)}$ & RCT, Double-blinded & 8 & 143 & 3.8 & 55 & 38.5 & Haloperidol \\
\hline & Weiden et al. $^{18)}$ & RCT, Open-label & 12 & 11 & 4.0 & 0 & 0.0 & Risperidone LAI \\
\hline & Mean/Total & & 32 & 274 & 3.9 & 88 & 24.5 & \\
\hline \multirow{4}{*}{$\begin{array}{l}\text { Risperidone } \\
\text { LAl }\end{array}$} & Weiden et $a l^{18)}$ & RCT, Open-label & 12 & 19 & 28.9 & 0 & 0.0 & Risperidone (Oral) \\
\hline & Emsley et al. ${ }^{12)}$ & Open-label & 104 & 50 & 32.8 & 14 & 28.0 & None \\
\hline & Kim et $a l^{17)}$ & RCT, Open-label & 104 & 25 & 29.0 & 3 & 12.0 & Risperidone (Oral) \\
\hline & Mean/Total & & 73 & 94 & 30.2 & 17 & 13.3 & \\
\hline Ziprasidone & Kahn et al. ${ }^{3)}$ & Open-label & 52 & 82 & 107.2 & 31 & 37.8 & Multiple \\
\hline ALL & Mean/Total & & 35.1 & 648 & & 190 & 29.3 & \\
\hline
\end{tabular}

SGA, second-generation antipsychotic; RCT, randomized-controlled trial; LAl, long-acting injectable.

table. No studies of amisulpiride, asenapine, iloperidone, lurasidone, paliperidone, or ziprasidone in FEP were identified. For comparative purposes, we have included data on all-causes discontinuation for both amisulpiride and ziprasidone from the EUFEST trial ${ }^{3)}$-the only published study of these agents in FEP-which was included in our previous review.

For each SGA as well as all trials combined, we calculated the mean all-causes discontinuation rate and the mean study duration. The mean SGA dose was also recorded for each trial. The data on all-causes discontinuation are also summarized in Table 1. The overall all-causes discontinuation rate was $29.3 \%$ and the mean duration of all studies was 35.1 weeks. The mean all-causes discontinuation rate was lowest for aripiprazole $(11.1 \%, \mathrm{n}=1$ study) and highest for quetiapine ( $50.5 \%, \mathrm{n}=3$ studies $)$. Of note, long-acting injectable risperidone had the longest mean study duration (73 weeks), but a lower mean all-causes discontinuation rate $(13.3 \%, n=3$ studies $)$ than all oral SGAs except for aripiprazole.

\section{LIMITATIONS AND COMPARISONS TO PREVIOUS FINDINGS}

There are several broad limitations of our methodology for describing all-causes discontinuation. For example, it might be expected that discontinuation rates would increase in proportion to the duration of the trial (i.e., higher all-causes discontinuation rates would be expected in longer trials), although, interestingly, this was not the case for the trials of long-acting injectable risperidone. However, in general, the small number of studies limited the ability to analyze for such a trend. Furthermore, it was also not possible to control for factors such as the number of trials for each SGA, patient demographics, study design (randomized versus open-label), medication dose (flexible versus fixed), and the option to switch medications. Thus, our findings should be interpreted with caution in light of study heterogeneity. Despite these inherent limitations, even this "crude" estimate provides useful information, and it is reassuring that our result is consistent with the prevalence reported in our previous review $(40.8 \%)^{5)}$ as well as in the EUFEST study $(42 \%){ }^{3)}$ which was a longer trial (52 weeks) than the mean duration in this report (35.1 weeks).

Furthermore, for the studies of long-acting injectable risperidone, subjects were treated with oral SGAs for one to twelve weeks prior to study entry. For example, in the study by Kim et al., ${ }^{17)}$ participants were stable on oral SGAs for 4 weeks prior to the baseline visit. Similarly, in the PREvent First Episode Relapse (PREFER) trial by Weiden et al., ${ }^{18)}$ subjects were treated for up to twelve weeks prior to randomization. Subjects randomized to long-acting injectable risperidone in this trial were given this recommendation in the context of a two-session psychoeducational intervention tailored to patients with FEP 
and their families. Thus, the potential for selection bias-towards patients who were both early responders to SGAs and better engaged in treatment-may have contributed to the lower all-causes discontinuation rate for this agent.

Although aripiprazole had the lowest all-causes discontinuation rate of all agents studied, we note the limitation that this finding was based on one small trial of relatively short duration. ${ }^{15)}$ Thus, future studies are needed to replicate this observation.

The all-causes discontinuation rates for oral risperidone $(24.5 \%)$ and olanzapine $(20.5 \%)$ were similar to each other, and these two SGAs also had similar discontinuation rates in our previous study. ${ }^{5}$ All-causes discontinuation rates for oral risperidone and olanzapine were lower than those for amisulpiride (30.8\%) and ziprasidone (37.8) from the EUFEST trial. ${ }^{3)}$ The TMAP notes that dosing for ziprasidone has not been systematically studied in FEP. The mean dose of ziprasidone in the EUFEST study was $107.2 \mathrm{mg} /$ day. One possible explanation for the relatively higher discontinuation rate for ziprasidone is that higher dosing is required in FEP.

Quetiapine had the highest all-causes discontinuation rate in both studies. Of note, both TMAP and PORT recommended higher doses of quetiapine (up to 500-600 $\mathrm{mg} /$ day) relative to doses of other SGAs. However, the mean dose of quetiapine in the four trials reviewed here was $400 \mathrm{mg} /$ day. For example, Berger et al ${ }^{11)}$ compared quetiapine 200 versus $400 \mathrm{mg}$ /day in a fixed dose trial. Patients randomized to the lower ( $200 \mathrm{mg} /$ day) dose were more likely to discontinue study medication due to both lack of efficacy and medication nonadherence (for which lack of efficacy may have also been a contributing factor). Thus, under-dosing of quetiapine may have contributed to the higher all-causes discontinuation rate for this agent.

\section{REASONS FOR MEDICATION DISCONTINUATION IN FEP}

A detailed analysis of specific causes of discontinuation was not feasible, owing to the heterogeneity mentioned above as well as the lack of this information in many studies. However, within individual studies, the incidence of several specific reasons for discontinuation is notable. Leading causes of discontinuation reported in specific trials are described below.

\section{Lack of Efficacy}

In the EUFEST study, Kahn et al. ${ }^{3)}$ found that $10.6 \%$ $(11 / 104)$ of patients randomized to amisulpiride and
$20.7 \%(17 / 82)$ of patients randomized to ziprasidone discontinued medication due to a lack of efficacy.

Emsley et al. ${ }^{12)}$ found that 21\% (3/14) of patients randomized to oral risperidone discontinued medication due to insufficient response, while Möller et al. ${ }^{13)}$ found a lower percentage of patients discontinued risperidone due to insufficient response $(13 \% ; 7 / 55)$. In the Esquire Trial, Gafoor et $_{\text {al. }}{ }^{16)}$ found an all-cause discontinuation rate for quetiapine of $73.7 \%$ versus $55.9 \%$ for oral risperidone. Although data on specific reasons for discontinuation were not reported, the authors noted that perceived lack of efficacy by both the patient and physician was the most common reason for discontinuation. In a study of the cognitive effects of treatment with quetiapine in antipsychotic-naïve patients with FEP, $6.5 \%$ of patients (3/46) discontinued medication because of lack of efficacy. ${ }^{10}$ ) Dosages of quetiapine used in this study were comparable to those used in the CAFÉ and EUFEST studies. Berger et al. ${ }^{11)}$ compared quetiapine in doses of $200 \mathrm{mg}$ versus 400 mg per day in patients with FEP. They found that $10.6 \%$ of patients (15/141) discontinued quetiapine due to lack of efficacy: 9 (14.5\%) patients from the $200 \mathrm{mg}$ group and 6 (9.4\%) patients from the $400 \mathrm{mg}$ group. They also noted that while the $200 \mathrm{mg}$ dose group had higher initial response rates, the $400 \mathrm{mg}$ dose group had higher rates of remission at the end of the trial. Thus, particularly for quetiapine, ensuring adequate dosing may be an important factor to improve rates of discontinuation.

\section{Intolerable Side Effects}

In the EUFEST study, $11.5 \%$ (12/104) of patients taking amisulpiride and $8.5 \%(7 / 82)$ of patients taking ziprasidone discontinued study medication due to adverse side effects. ${ }^{3)}$

Möller et al. ${ }^{13)}$ found that 25\% (14/55) of patients taking oral risperidone discontinued medication secondary to side effects. The most common side effects leading to discontinuation were EPS $(n=4 ; 7 \%)$, depression, suicidal tendency, or somatic disorder accounted for $(n=4 ; 7 \%)$, and other side effects $(\mathrm{n}=6 ; 11 \%)$.

Takahashi et al. ${ }^{14)}$ found that both patients taking aripiprazole who discontinued medication did so due to side effects, specifically worsening psychotic symptoms and insomnia.

Andersen et al ${ }^{10)}$ found that $8.7 \%$ of patients $(4 / 46)$ discontinued treatment with quetiapine due to side effects, although specific side effects were not described. In the study by Berger et al. ${ }^{11)} 2.8 \%$ of patients (4/141) discontinued treatment with quetiapine due to side effects, 
most commonly sedation, altered sleep, concentration difficulties, asthenia, depression, and orthostatic dizziness. Also of note was that tremor and reduced salivation were both more frequently reported in the $400 \mathrm{mg}$ dose group, and thus were determined to be dose-dependent.

As outlined in the PORT recommendations, ${ }^{6)}$ four major classes of potential side effects of SGAs are noted: weight gain and associated metabolic effects, prolactin elevation and associated sexual side effects, QTc prolongations, and extrapyramidal symptoms, including tardive dyskinesia. Thus, careful consideration of patients' liabilities to certain side effects when selecting an SGA for treatment of FEP may help decreased the likelihood of medication discontinuation.

\section{Medication Non-Adherence}

In the EUFEST study, non-compliance was cited as the reason for medication discontinuation for $8.7 \%$ (9/104) of patients taking amisulpiride and $8.5 \%$ (7/87) of patients taking ziprasidone. ${ }^{3)}$ Möller et al. ${ }^{13)}$ found medication non-adherence was cited as the reason for discontinuation of $28 \%(15 / 55)$ of patients taking risperidone.

For quetiapine, Andersen et al. ${ }^{10)}$ reported that $30.4 \%$ of patients (14/46) were noncompliant with treatment (either medication or cognitive testing), and Berger et al. ${ }^{11)}$ reported noncompliance with treatment in 5.0\% of patients (7/141). The etiology of medication nonadherence is likely multifactorial, and adherence behavior should be routinely addressed in treatment.

\section{Patient Decision}

In two large studies of $\mathrm{FEP}^{1,2)}$ the patient's decision to discontinue medication against the preferences of the treating clinician was a common reason for discontinuation, occurring in $22.9 \%$ of patients $(30 / 131)$ on olanzapine in the study by Green et al. ${ }^{1)}$ and $41.5 \%$ of all patients (166/400) in the CAFE study. ${ }^{2}$ )

Cuesta et al. ${ }^{15)}$ examined cognitive effects of olanzapine and risperidone, and reported a $50.0 \%$ all-cause discontinuation rate (22/44 total discontinuations). $20.5 \%$ of discontinuations were due to patient withdrawals during the course of the study; however, no further evaluation of reasons for discontinuation was provided. Berger et al. ${ }^{11)}$ reported that $3.5 \%$ of patients $(5 / 141)$ withdrew consent during the study. These findings suggest a potential area for further exploration to improve medication adherence.

\section{RISK FACTORS FOR MEDICATION DISCONTINUATION IN FEP}

As previously reviewed, ${ }^{5)}$ replicated risk factors for medication discontinuation in FEP include severity of psychopathology, impaired insight into illness, negative patient attitudes towards medication, comorbid substance use, poor social support, and other barriers such as inability to afford medications. Several recent studies have added to this body of knowledge.

In a sample of $n=228$ patients with FEP from the EUFEST trial, Gaebel et al. ${ }^{19)}$ found that the Drug Attitude Inventory (DAI) was a significant, valid predictor of efficacy, as measured by treatment discontinuation. Thus, completion of this self-report instrument by patients early in treatment may help identify patients at heightened risk for medication discontinuation. Further studies of the use of this instrument in FEP are warranted.

The CAFÉ study group ${ }^{20)}$ also analyzed risk factors for medication discontinuation against medical advice, and found that poor treatment response and poor medication adherence were both significant independent risk factors. Furthermore, poor medication adherence was strongly predicted by ongoing substance abuse, ongoing depression, and treatment response failure. Poor adherence was also associated with black ethnicity, higher baseline cognitive performance, and reaching remission status. The authors did not, however, find medication side effects to be a risk factor for poor medication adherence or discontinuation, and could not find a reason for the association between higher baseline cognitive performance and poor medication adherence. Taken together, these findings suggest that thorough assessment of risk factors for discontinuation, response to treatment, and monitoring of side effects and attitudes towards medication may improve discontinuation rates. Further investigation of predictive factors for medication discontinuation is warranted.

\section{INTERVENTIONS FOR IMPROVING MEDICATION DISCONTINUATION RATES IN FEP}

In a trial of oral versus long-acting injectable risperidone, Weiden et al. ${ }^{18)}$ commented that for most patients with FEP "the central adherence question is not 'Will this patient stay on medication?' but rather 'How long will this patient stay on medication before stopping?" Certainly our findings are in accord with this very sobering 
statement. Given the scope and implications of medication discontinuation in FEP-including a decreased likelihood of remission, ${ }^{21)}$ a five-fold increased risk of relaps $\mathrm{e}^{22,23)}$ and an increased probability of treatment-resistant symptoms ${ }^{22,24-26)}$ and decreased overall outcome $\mathrm{e}^{27)}$-there is clearly a need to identify effective interventions to improve adherence in this patient population.

In patients with chronic psychosis, there is varying evidence for improved adherence with several interventions, including the use of particular drug formulations such as long-acting injections and orally disintegrated tablets, psychotherapy, peer support services, and family support/interventions. However, such interventions are understudied in FEP. We highlight here two approaches with evidence of success in FEP.

\section{Relapse Prevention Therapy}

In the Episode II trial, Gleeson et al. ${ }^{28)}$ performed a randomized controlled trial comparing the effectiveness of relapse prevention therapy (RPT) versus treatment as usual (TAU) in a specialist FEP service, with antipsychotic medication and group-based psychosocial interventions as background treatments in both conditions. RPT included a multimodal individual and family-based psychosocial intervention in patients age 15-25 years following remission of FEP. Individual therapy involved 5 phases within a cognitive-behavioral therapy framework as follows: (1) Engaging the patient and assessing their extent of recovery and risk for relapse; (2) Therapist and patient agreement on a formulation and agenda for therapy which was summarized in a letter and read out loud to the patient; (3) Increasing awareness for the risk of setbacks and how to minimize them; (4) Identification of early warning signs of relapse and the formulation of a relapse plan; and (5) additional optional modules addressing issues including nonadherence to treatment, substance abuse, coping skills, and comorbid anxiety and depression. The family component included assessment of family communication, burden, and coping, psychoeducation about relapse risk, and discussion of early warning signs of relapse and review of the relapse prevention plan. They found significantly higher relapse rates in the TAU group versus the RPT group ( $21.8 \%$ versus $5.3 \%$, respectively), and that relapses occurred significantly earlier in the TAU than in the RPT group (on average 241 days versus 273 days). The authors also found that the efficacy of RPT was not better accounted for by medication adherence or severity of substance abuse, suggesting that RPT has a spe- cific effect in reducing relapse risk.

\section{Long-acting Injectable Agents}

Another potential approach to improving discontinuation rates in FEP is the use of long-acting injectable agents. While these drugs are classically reserved for patients with chronic schizophrenia at high-risk for noncompliance, findings from our review suggest that these agents are acceptable and effective in FEP. Kim et al. ${ }^{17}$ explored the effectiveness of risperidone long-acting injection (RLAI) in FEP by comparing medication adherence and relapse rates. Medication adherence scores were significantly higher in the RLAI group than in the oral risperidone group, and the RLAI group showed significantly lower one and two year relapse rates. $97 \%$ of patients achieving remission stayed in remission until study completion (104 weeks) and remission was associated with greater improvement in multiple symptom domains, insight, and social and occupational functioning. Emsley et $a .^{12)}$ assessed rates, predictors, and correlates of remission in patients with FEP treated with RLAI using the 'Remission in Schizophrenia Working Group' criteria. A remission rate of $64 \%$ was achieved, $97 \%$ of who had sustained remission until completion of the study (104 weeks). Weiden et al. ${ }^{18)}$ compared initial adherence with RLAI versus continuation on oral atypical antipsychotics during FEP. $73 \%$ of patients randomized to RLAI accepted the recommendation and received their initial injection 6 weeks after randomization. Almost all of RLAI acceptors expressed initial reluctance to convert from oral medication to RLAI; however, subjects generally accepted the recommendation after completing a two-session psychoeducational intervention tailored to first-episode patients and their families. The sessions presented recommended RLAI within the framework of a life-goals approach with emphasis on voluntary acceptance, and the authors concluded the commonly held clinical belief that first-episode patients would never accept a long acting injection is untrue. Taken together, the trials support the utility and efficacy of long-acting injectable risperidone early in the course of illness, which have a discontinuation rate that is lower than many oral agents.

\section{MEDICATION DISCONTINUATION FOLLOWING REMISSION IN FEP}

In addition to the issue of medication discontinuation in the acute treatment of FEP, another equally important question is the optimal duration of maintenance anti- 
psychotic treatment for patients in remission following a FEP. This decision requires consideration of multiple factors, including response to treatment, patient preferences, risk of relapse, and risk of metabolic disturbances and/or movement disorders.

The TMAP for schizophrenia notes "a trial period off antipsychotics may be reasonable for some patients early in the course of illness. This, an individualized decision, depends on a number of factors that do not lend themselves to an algorithmic approach. Thus, the schizophrenia algorithm contains no guidelines for antipsychotic medication discontinuation, which is anticipated to be a rare event in the typical mental health clinic patient population." "7) Although treatment guidelines generally recommend at least 1 year of antipsychotic treatment, some consider indefinite maintenance treatment reasonable. The American Psychiatric Association Practice Guideline for the Treatment of Patients with Schizophrenia, Second Edition concluded, "Unfortunately there is no reliable indicator to differentiate the minority who will not from the majority who will relapse with drug discontinuation. Indefinite maintenance antipsychotic medication is recommended for patients who have had multiple prior episodes or two episodes within five years." $^{29)}$

At present, there are currently no consensus guidelines for how medication can be optimally discontinued in patients in remission from FEP. Wang et al. ${ }^{30)}$ investigators sought to determine length of maintenance treatment needed with an initial therapeutic dose of risperidone, in contrast to a reduction to a lower dose, following stabilization of the acute phase of schizophrenia in three groups. Patients were randomized to the 4-week, 26-week, and no-dose reduction groups. Patients in the 4-week group were maintained with their initial therapeutic risperidone dose for 4 weeks. The dose was then decreased by $0.5 \mathrm{mg}$ every 7-10 days (depending on the starting dose) to a target of one-half of the initial therapeutic dose over the next 8 weeks, which was maintained until completion of the study. Patients in the 26-week group were maintained with initial therapeutic risperidone dose for 26 weeks, and then gradually reduced in the same manner as the 4-week group to one-half of the therapeutic dose, which was maintained until completion of the study. In the no-dose reduction group, patients were maintained on full initial therapeutic dose of risperidone throughout the study. Relapse rates were $24.0 \%, 15.8 \%$, and $7.8 \%$ for the 4 -week, 26 -week, and no-dose reduction groups, respectively. The authors found that shorter periods of administration of therapeutic risperidone doses were associated with increased risk and earlier relapse, but not with significantly lower risk of adverse effects. They determined that at least one year of maintenance risperidone treatment at the initial therapeutic dose is safe and more effective in relapse prevention than reduced dose strategies.

A study by Wunderink et al. ${ }^{31)}$ examined guided discontinuation of antipsychotic treatment in 131 patients ages 18 to 45 year who had achieved remission of FEP for at least 6 months. The study was a prospective 2-year trial in which patients were randomized but openly assigned to maintenance treatment or the guided discontinuation strategy (raters were blinded throughout). Maintenance treatment was carried out using low-dose atypical antipsychotics as per the American Psychiatric Association guidelines, and guided discontinuation (over an average of 4.6 months) was carried out by gradual symptom-based tapering. Patients were followed for 18 months and relapse rates and social and vocational functioning were the primary outcome measures. Relapse rates were twice as high in the group receiving guided discontinuation $(43 \%$ vs. $21 \%$ ), and only $21.5 \%$ of the patients were successfully discontinued. In addition, $46.2 \%$ of patients were not able to have their medication discontinued, $24.6 \%$ restarted medication due to relapse, and $7.7 \%$ restarted medication due to mild recurrent symptoms without relapse. Therefore, while the authors found that a small proportion of patients who have achieved remission can be successfully tapered off of their antipsychotic medication, this may not be universally applicable.

\section{CONCLUSIONS}

Medication discontinuation is a major treatment issue in FEP right from the onset of treatment. Similar to our previous results, we found that almost $30 \%$ of patients with FEP discontinue medication in the first 9 months of treatment, a finding that has important implications for long-term outcomes. Only olanzapine, quetiapine, and risperidone (oral and long-acting injectable) have been evaluated in multiple trials in FEP. Further study of newer agents is warranted, as effective dose ranges for specific agents may be different in FEP versus chronic psychosis. The self-reported Drug Attitude Inventory may help identify patients at heightened risk for medication discontinuation. In addition to vigilant monitoring for and adequate treatment of psychopathology and medication side effects, Relapse Prevention Therapy and the use of longacting injectable agents may decrease discontinuation 
rates in FEP. There is currently no consensus on how long a patient should remain on an antipsychotic medication following remission of FEP. Studies are needed to identify predictors of which patients in remission from FEP are less likely to relapse when medication is discontinued.

Taken together, our findings presented here underscore the importance of addressing medication discontinuation both as a means of preventing long-term morbidity and enhancing remission and functional recovery in FEP.

\section{REFERENCES}

1. Green AI, Lieberman JA, Hamer RM, Glick ID, Gur RE, Kahn RS, et al. Olanzapine and haloperidol in first episode psychosis: two-year data. Schizophr Res 2006;86:234-243.

2. McEvoy JP, Lieberman JA, Perkins DO, Hamer RM, Gu H, Lazarus A, et al. Efficacy and tolerability of olanzapine, quetiapine, and risperidone in the treatment of early psychosis: a randomized, double-blind 52-week comparison. Am J Psychiatry 2007;164:1050-1060.

3. Kahn RS, Fleischhacker WW, Boter H, Davidson M, Vergouwe Y, Keet IP, et al. Effectiveness of antipsychotic drugs in first-episode schizophrenia and schizophreniform disorder: an open randomised clinical trial. Lancet 2008; 371:1085-1097.

4. Lieberman JA, Stroup TS, McEvoy JP, Swartz MS, Rosenheck RA, Perkins DO, et al. Effectiveness of antipsychotic drugs in patients with chronic schizophrenia. $N$ Engl $J$ Med 2005;353:1209-1223.

5. Miller BJ. A review of second-generation antipsychotic discontinuation in first-episode psychosis. $J$ Psychiatr Pract 2008;14:289-300.

6. Buchanan RW, Kreyenbuhl J, Kelly DL, Noel JM, Boggs DL, Fischer BA, et al. The 2009 schizophrenia PORT psychopharmacological treatment recommendations and summary statements. Schizophr Bull 2010;36:71-93.

7. Moore TA, Buchanan RW, Buckley PF, Chiles JA, Conley RR, Crismon ML, et al. The Texas Medication Algorithm Project antipsychotic algorithm for schizophrenia: 2006 update. J Clin Psychiatry 2007;68:1751-1762.

8. Lieberman JA, Phillips M, Gu H, Stroup S, Zhang P, Kong $\mathrm{L}$, et al. Atypical and conventional antipsychotic drugs in treatment-naive first-episode schizophrenia: a 52-week randomized trial of clozapine vs chlorpromazine. Neuropsychopharmacology 2003;28:995-1003.

9. Schooler N, Rabinowitz J, Davidson M, Emsley R, Harvey $\mathrm{PD}$, Kopala L, et al. Risperidone and haloperidol in firstepisode psychosis: a long-term randomized trial. Am J Psychiatry 2005;162:947-953.

10. Andersen R, Fagerlund B, Rasmussen H, Ebdrup BH, Aggernaes B, Gade A, et al. Cognitive effects of six months of treatment with quetiapine in antipsychotic-naïve firstepisode schizophrenia. Psychiatry Res 2011;187:49-54.

11. Berger GE, Proffitt TM, McConchie M, Kerr M, Markulev $\mathrm{C}$, Yuen HP, et al. Dosing quetiapine in drug-naive first-episode psychosis: a controlled, double-blind, randomized, single-center study investigating efficacy, tolerability, and safety of $200 \mathrm{mg} /$ day vs. $400 \mathrm{mg} /$ day of quetiapine fumarate in 141 patients aged 15 to 25 years. J Clin Psychiatry 2008;69:1702-1714.

12. Emsley R, Oosthuizen P, Koen L, Niehaus DJ, Medori R, Rabinowitz J. Remission in patients with first-episode schi- zophrenia receiving assured antipsychotic medication: a study with risperidone long-acting injection. Int Clin Psychopharmacol 2008;23:325-331.

13. Möller HJ, Riedel M, Jäger M, Wickelmaier F, Maier W, Kühn KU, et al. Short-term treatment with risperidone or haloperidol in first-episode schizophrenia: 8-week results of a randomized controlled trial within the German Research Network on Schizophrenia. Int J Neuropsychopharmacol 2008;11:985-997.

14. Takahashi H, Oshimo T, Ishigooka J. Efficacy and tolerability of aripiprazole in first-episode drug-naive patients with schizophrenia: an open-label trial. Clin Neuropharmacol 2009;32:149-150.

15. Cuesta MJ, Jalón EG, Campos MS, Peralta V. Cognitive effectiveness of olanzapine and risperidone in first-episode psychosis. Br J Psychiatry 2009;194:439-445.

16. Gafoor R, Landau S, Craig TK, Elanjithara T, Power P, McGuire P. Esquire trial: efficacy and adverse effects of quetiapine versus risperidone in first-episode schizophrenia. J Clin Psychopharmacol 2010;30:600-606.

17. Kim B, Lee SH, Choi TK, Suh S, Kim YW, Lee E, et al. Effectiveness of risperidone long-acting injection in firstepisode schizophrenia: in naturalistic setting. Prog Neuropsychopharmacol Biol Psychiatry 2008;32:1231-1235.

18. Weiden PJ, Schooler NR, Weedon JC, Elmouchtari A, Sunakawa A, Goldfinger SM. A randomized controlled trial of long-acting injectable risperidone vs continuation on oral atypical antipsychotics for first-episode schizophrenia patients: initial adherence outcome. J Clin Psychiatry 2009;70: 1397-1406.

19. Gaebel W, Riesbeck M, von Wilmsdorff M, Burns T, Derks EM, Kahn RS, et al. Drug attitude as predictor for effectiveness in first-episode schizophrenia: Results of an open randomized trial (EUFEST). Eur Neuropsychopharmacol 2010;20:310-316.

20. Malla A, Norman R, Schmitz N, Manchanda R, BéchardEvans L, Takhar J, et al. Predictors of rate and time to remission in first-episode psychosis: a two-year outcome study. Psychol Med 2006;36:649-658.

21. Perkins DO, Gu H, Weiden PJ, McEvoy JP, Hamer RM, Lieberman JA. Comparison of Atypicals in First Episode study group. Predictors of treatment discontinuation and medication nonadherence in patients recovering from a first episode of schizophrenia, schizophreniform disorder, or schizoaffective disorder: a randomized, double-blind, flexible-dose, multicenter study. J Clin Psychiatry 2008;69:106113.

22. Robinson D, Woerner MG, Alvir JM, Bilder R, Goldman R, Geisler S, et al. Predictors of relapse following response from a first episode of schizophrenia or schizoaffective disorder. Arch Gen Psychiatry 1999;56:241-247.

23. Rabiner CJ, Wegner JT, Kane JM. Outcome study of first-episode psychosis. I: Relapse rates after 1 year. Am J Psychiatry 1986;143:1155-1158.

24. Gitlin M, Nuechterlein K, Subotnik KL, Ventura J, Mintz $\mathrm{J}$, Fogelson DL, et al. Clinical outcome following neuroleptic discontinuation in patients with remitted recent-onset schizophrenia. Am J Psychiatry 2001;158:1835-1842.

25. Lieberman JA, Perkins D, Belger A, Chakos M, Jarskog F, Boteva $\mathrm{K}$, et al. The early stages of schizophrenia: speculations on pathogenesis, pathophysiology, and therapeutic approaches. Biol Psychiatry 2001;50:884-897.

26. Robinson DG, Woerner MG, Alvir JM, Geisler S, Koreen A, Sheitman B, et al. Predictors of treatment response from a first episode of schizophrenia or schizoaffective disorder. 
Am J Psychiatry 1999;156:544-549.

27. Bachmann S, Bottmer C, Schroder J. One-year outcome and its prediction in first-episode schizophrenia-a naturalistic study. Psychopathology 2008;41:115-123.

28. Gleeson JF, Alvarez-Jimenez M, Cotton SM, Parker AG, Hetrick S. A systematic review of relapse measurement in randomized controlled trials of relapse prevention in first-episode psychosis. Schizophr Res 2010;119:79-88.

29. Lehman AF, Lieberman JA, Dixon LB, McGlashan TH, Miller AL, Perkins DO, et al. Practice guideline for the treatment of patients with schizophrenia, second edition. Am J Psychiatry 2004;161(2 Suppl):1-56.

30. Wang CY, Xiang YT, Cai ZJ, Weng YZ, Bo QJ, Zhao JP, et al. Risperidone maintenance treatment in schizophrenia: a randomized, controlled trial. Am J Psychiatry 2010;167: 676-685.

31. Wunderink L, Nienhuis FJ, Sytema S, Slooff CJ, Knegtering $\mathrm{R}$, Wiersma D. Guided discontinuation versus maintenance treatment in remitted first-episode psychosis: relapse rates and functional outcome. J Clin Psychiatry 2007;68:654-661. 